\title{
Las escuelas rurales de La Habana: un problema sin resolver en la República Neocolonial entre 1936 y 1952
}

\author{
Yusdiel Leon Castillo ${ }^{1}$ \\ ${ }^{1}$ Universidad de Artemisa. Departamento de Marxismo, Leninismo e Historia. Calle 8C No 713 e/7 y campo M- \\ 22 zona urbana Consejo Popular Lincoln 33800. Artemisa, Cuba. yusdiellc@uart.edu.cu.
}

RESUMEN. En Cuba existe un vacío historiográfico respecto a las escuelas rurales de La Habana entre 1936 y 1952, por lo que se plantea como problema de investigación la manifestación de las condiciones materiales y humanas de esas escuelas en la solución de los problemas educativos de la enseñanza cubana durante esos años. El objetivo general de este artículo es caracterizar las condiciones materiales y psicológicas en las que los maestros debían desarrollar sus clases. Fueron empleados métodos del nivel teórico y empírico respectivamente, tales como: histórico-lógico, analítico-sintético, inductivo-deductivo y el análisis documental. Entre los principales resultados se encuentra que los planes de estudio y programas de las escuelas rurales de La Habana favorecieron la solución de algunos de los principales problemas educativos existentes en la enseñanza, sin embargo varias de las regulaciones que estos planteaban no alcanzaron a concretarse en toda su amplitud debido a la desatención de los gobiernos republicanos.

Palabras clave: Escuelas Rurales, Maestros Rurales, Programas, Prácticas Educativas. 


\title{
The rural schools of Havana: a problem without turns into the Neo-colonial republic between 1936 and 1952
}

\begin{abstract}
In Cuba, exist a historiographical vacuum respect to the rural schools of La Havana between 1936 and 1952, for which it outlines as problem of investigation the manifestation of the material conditions and it humanize of those schools in the solution of the educational problems of the Cuban teaching during those years. The general objective of this article is to characterize the material and psychological conditions in those who the teachers must develop your classes. They were employed methods of the theoretical and empiric level respectively, just as historical, logical, analytic, synthetic, and inductive-deductive and the documentary analysis. Between the main results, it finds that the plains of study and program of the rural schools of La Havana favored the solution of one of the main educational existent problems in the teaching, however several of the regulations that these outlined did not manage to make concrete in all your amplitude due to the inattention of the republican governments.
\end{abstract}

Keywords: Rural Schools, Rural Teachers, Program, Practical Educational. 


\section{As escolas rurais de Havana: um problema sem resolver na República Neocolonial de 1936 a 1952}

RESUMO. Em Cuba há um vazio historiográfico no que diz respeito às escolas rurais em Havana de 1936 a 1952, se caracterizando como problema de pesquisa a manifestação de condições materiais e humanas dessas escolas na resolução de problemas educacionais da educação cubana durante esses anos. Nesta pesquisa científica é apresentado como objetivo principal caracterizar as condições materiais e psicológicas em que os professores desenvolvem as suas aulas. Foram utilizados métodos de nível teórico e empírico, respectivamente, tais como: histórico e lógico, analítico-sintético, indutivo-dedutivo e estudo documental. Entre os principais resultados foi constatado que os currículos e programas de escolas rurais em Havana apresentam soluções para alguns problemas educacionais existentes no ensino ofertado por essas instituições; no entanto, vários dos regulamentos que essas escolas implantaram não foram capazes de materializar todo a amplitude do ensino devido à negligência dos governos republicanos, embora as suas contribuições para a pedagogia e didática tenham sido significativas.

Palavras-chave: Escolas Rurais, Professores Rurais, Programas, Práticas Educacionais. 


\section{Introducción}

La educación en la sociedad tiene una vinculación profunda con todas las estructuras sociales, y permite el desarrollo de los ciudadanos que la conforman. Este proceso educativo constituye un elemento de importancia en la conformación de la identidad nacional, a partir de estudiar el pensamiento pedagógico que se generó en la sociedad cubana, en especial en la república neocolonial, como forma de resistencia y de rescate de una tradición en el país. Para ello, resulta necesario historiar los diferentes períodos por los que ha transitado la educación en Cuba y en especial el pensamiento pedagógico de sus educadores.

Diversas indagaciones afirman que constituye una tarea aún pendiente en la historiografía cubana el estudio del período republicano (1902-1958), pues existen temáticas que requieren ser profundizadas por la insuficiencia de su tratamiento en diversas investigaciones. Entre las líneas que resultan carentes en los diferentes estudios se encuentran las escuelas rurales entre los años 1936 y 1952, sobre todo su modo de existir en La Habana, debido a las características del territorio y a las complejidades educativas dadas por la multiplicidad de escuelas que coexistían: la urbana pública, la urbana privada, los colegios religiosos y la rural. Se debe tener en cuenta que esta provincia era el centro del desarrollo económico, político, social y cultural del país, que además estuvo marcada por la efervescencia revolucionaria legada por la Revolución de 1930 y por la huelga revolucionaria de marzo de 1935, en la que los maestros jugaron un papel determinante.

El estudio de las escuelas rurales de La Habana entre 1936 y 1952 presupone atender a las características sociopolíticas, organizativas, curriculares y metodológicas que sirvieron de marco a su evolución. Igualmente exige contextualizar esta problemática en la localidad objeto de trabajo, teniendo en consideración su desarrollo en la nación.

El análisis de la temática precisa recurrir a una amplia bibliografía que brinda el sostén adecuado a la investigación y posibilita un mayor conocimiento y profundidad de las ideas a abordar, por lo que se propone como objetivo general de este artículo caracterizar las condiciones materiales y psicológicas en las que los maestros debían desarrollar sus clases en el período republicano de 1936 a 1952, teniendo en cuenta los antecedentes históricos de esta temática.

Como año inicial de la investigación se escoge el 1936, ya que marcó un auge en las escuelas rurales con aprobación y 
posterior instrumentación del Decreto Ley No. 620 del 27 de febrero de ese año, por el gobierno provisional de José A. Barnet Vinajeras. Leonardo Anaya Murillo fungía como Secretario de Educación y Fulgencio Batista como Coronel Jefe del Ejército Constitucional. Se evidenció una evolución favorable de la escuela rural cívico-militar.

La ley autorizaba al Jefe del Ejército permitirles a los miembros de la institución a prestar servicios de enseñanza primaria (teniendo en cuenta que el objetivo fundamental del proyecto era alfabetizar) en los lugares donde no existieran escuelas. Luego se hizo extensivo el permiso a efectivos de la Marina de Guerra y a la Policía.

Con esta ley no solo se beneficiaron las escuelas rurales cívico-militares que surgieron, sino que también para las ya existentes significó una renovación en algunas de sus infraestructuras, en sus concepciones y en sus métodos de trabajo.

El año 1952 marca una ruptura abrupta en el abandono a las escuelas rurales. Si bien la Constitución de 1940 en los artículos 51 y 52 respaldaba que la enseñanza pública se constituiría de forma orgánica y sería dotada en los presupuestos del Estado, esto no se materializó en la práctica educativa, pues las escuelas rurales bajo el gobierno de Carlos Prío Socarrás sufrían de insólitas condiciones higiénico-sanitarias. Esto significó un abandono total, a partir de la instauración del gobierno inconstitucional de Fulgencio Batista.

\section{Desarrollo}

En el estado incipiente y caótico de la instrucción popular de Cuba en la etapa colonial, pretender encontrar escuelas en las comarcas rurales del territorio que ocupaba La Habana, sería una pretensión tan quimérica como atribuirles existencia y eficacia reales a las escuelas para indígenas y negros esclavos que algunas Leyes de Indias ordenaron establecer. Las comarcas rurales solo tuvieron doctrineros $\mathrm{O}$ profesores de enseñanza ambulantes que tenían licencia para ocuparse en dar lecciones en las haciendas y caseríos, sin otro requisito previo que resignarse con los reducidos haberes que su trabajo les produjera, por lo que es presumible que fuese muy reducido el número de ellos.

Aún en los comienzos del siglo XIX persistía la inexistencia de escuelas rurales, pues si bien es cierto que la Real Sociedad Económica de Amigos del País pretendió establecerlas, un informe de esta Corporación demuestra que hasta el año 1800 no se había podido hacer ni conseguir. En el año 1819, la Sección de Educación de la Real Sociedad no obtuvo la formación de las Juntas Rurales para los 
partidos del campo, compuestas en cada uno, por el párroco, el juez pedáneo y dos vecinos notables.

El cometido de las Juntas Rurales era la creación, sostenimiento, mejora e inspección de las escuelas públicas de enseñanza primaria y gratuita en sus respectivas comarcas rurales para sacar a los habitantes de estas del estado de rusticidad en que se encontraban e inspirarles, por medio de la enseñanza, la búsqueda del conocimiento. Aunque no dejaron de establecerse algunas escuelas rurales en determinados caseríos y poblados.

En los pueblos cuyo número de vecinos excedía de quinientos, se establecieron dos escuelas, tres si pasaban de mil, y en general una más por cada quinientos vecinos.

Este fue el tipo de escuela rural concebido y creado al amparo de la primera legislación sobre enseñanza pública; escuela prácticamente inferior a la escuela primaria elemental que esa misma legislación concibió para los centros de población de relativa densidad, pues no solo limitó a lo indispensable las materias objeto de la enseñanza, sino que autorizó para que sirvieran las escuelas rurales a personas sin ninguna preparación ni competencias demostradas. La escuela rural que ocupaba el territorio habanero no estaba preparada para cumplir debidamente la triple finalidad, humanitaria, civilizadora y nacionalista a pesar de que hacía siete años regía el Plan General de Estudios de 27 de octubre de 1844, que disponía el establecimiento de escuelas con la más amplia liberalidad.

El día 15 de julio de 1863 fue promulgado un nuevo Plan de Estudios para la Isla de Cuba. Este plan fue más restrictivo con respecto al establecimiento de escuelas. Los artículos 7mo y 8vo del Plan anterior fueron sustituidos en el nuevo por dos Artículos, 173 y 174. El artículo 173 según planteaba Correoso del Risco, Felipe (1931):

El Gobernador Superior Civil, oyendo a los respectivos Ayuntamientos, determinará, en previo informe de la Junta Superior de Instrucción Pública, el número de escuelas públicas elemental de niños y nidos que deberá haber en cada poblado y en los partidos rurales.

Según el mismo autor en el nuevo Plan de Estudios de 1863, las escuelas rurales tampoco derivaron ningún beneficio, siquiera fuese en el propósito del gobernante de la Isla. El 7 de diciembre de 1880 fue promulgado un nuevo Plan de Instrucción Pública y último del período colonial, que había de regirse en el curso de 1882 y que tendía a promover el mejoramiento de la enseñanza. Los Artículos 125, 126 y 128 regulaban el 
establecimiento de escuelas en la forma siguiente:

Artículo 125: En todo pueblo de quinientas almas habrá necesariamente una, Escuela Pública elemental de niños y otra aunque sea incompleta de niñas.

Artículo 126: En los pueblos que lleguen a dos mil almas, habrá dos escuelas de niños y otras dos de niñas. En los que tengan cuatro mil almas, habrá tres y así sucesivamente aumentándose una escuela de cada sexo por cada dos mil habitantes.

La Legislación española planteaba la separación según sexos, lo que no propiciaba el desarrollo integral del niño, ni la educación con enfoque de género, además no atendía a un serio problema del campesinado cubano y en específico el habanero, atender a la diseminada población rural.

El gobierno colonial, ni cuando el presupuesto general alcanzó con veintinueve mil millones anuales, atendió al sostenimiento de la enseñanza primaria, dejando esa obligación íntegra a los Ayuntamientos, en su mayoría improductivos o en constante déficit, por lo que, siéndoles imposible pagarles regularmente a los maestros, les permitían legalmente el cobro de pensiones a los estudiantes, lo que hacía de la escuela pública rural, una escuela privada a la que se limitaba su asistencia por su pago.

Los padres de familias rurales, que lectura y escritura, pagaban a personas ancianas o inútiles para otros trabajos con una módica retribución y el alojamiento en la finca para que los instruyeran en esas materias.

Ante tal paradoja que establecía la propia legislación que creaba a las escuelas rurales y que a su vez la desnaturalizaba y la anulaba, existían criterios como el de Correoso (1931) plantea: La escuela rural cubana bajo la misma legislación que la creaba; por invalidarla y preterirla las realidades del medio ambiente, bajo la influencia de ideas y costumbres atávicas, no existió nunca en Cuba durante el período colonial.

El criterio de Correoso (1931) se comprueba al analizar las estadísticas por provincias sobre el analfabetismo de la población rural al cesar la dominación española el primero de enero de 1899. El territorio de La Habana contaba con una población rural de 163495 habitantes y de ellos 108108 no sabían firmar lo que significaba un 66 por ciento de analfabetismo que aunque era el más inferior de las provincias del país era una cifra significativa si se tiene en cuenta que era en esta donde había mayor desarrollo económico y social.

El campesino cubano, durante el período colonial, no dispuso de la escuela pública rural y gratuita que lo instruyera y deseaban que sus hijos aprendieran algo de 
que promoviera su mejoramiento $\mathrm{y}$ bienestar, a pesar de las leyes y disposiciones del gobierno metropolitano, así como de propósitos loables, que no pueden desconocerse ni olvidarse, que se producían en favor de la educación pública.

Durante el primer año del período de ocupación militar (1899) bajo las órdenes militares de John Rutter Brooke, funcionaban 313 escuelas rurales en todo el país; pero no fue hasta el primero de diciembre del propio año que entró en vigor la Orden Militar 221, del Cuartel General de la División de Cuba, con que se fundó la legislación interventora para las escuelas públicas cubanas.

La Orden citada se guió por lo porque establecí la Ley de Instrucción Pública española de 1880, revelando, además, la influencia norteamericana, pues solo es atribuible a estos antecedentes su tendencia a dar a los niños rurales una escuela diferente a la de los niños urbanos, no obstante el precepto contenido en su Artículo XXI hacía referencia a que todos los alumnos inscriptos tenían derecho a ser atendidos y educados de igual manera.

El sistema de escuelas públicas establecido por el Gobierno Interventor Norteamericano se desenvolvió rápidamente. En mayo de 1900 el número de aulas que funcionaban en Cuba había aumentado considerablemente.

Esto fue tan alentador que el Superintendente General de Escuelas de Cuija, el eminente pedagogo norteamericano Frye Alexis E., al dar a conocer a los maestros cubanos, por medio de la Circular No 9, la invitación que la Universidad de Harvard les dirigía para que concurrieran a su curso de verano del año 1900, justificaba tan insólita como bondadosa cortesía diciendo: El entusiasmo que ha demostrado el pueblo cubano, organizando sobre tres mil escuelas con ciento treinta mil pupilos, en el corto ejercicio de sesenta días.

En la mencionada circular, se borraron las jerarquías entre los maestros que servían las escuelas cubanas, fuesen urbanas o rurales; los sueldos se basaron en las exigencias de la vida de cada localidad y en la intensidad del trabajo, no en clase de escuela ni de enseñanza. Se pusieron las escuelas bajo una dirección uniforme, tanto en lo administrativo como en lo técnico; se elevó, en fin, la escuela rural al mismo plano de la escuela urbana, y en la división territorial que mantuvo de Distrito Urbanos y Distritos Municipales, ofreció a los intereses propios de las comarcas rurales garantía en las Juntas de Educación, integradas por padres. 
Los hechos anularon la elevada tendencia igualitaria de la legislación, pues a las escuelas rurales fueron destinados los maestros más jóvenes, inexpertos y con falta de preparación, restándoles así eficacia y prestigio, manteniéndolas de este modo en el plano de inferiores condiciones que siempre habían ocupado.

Luchas terribles, sañudas se suscitaron entre los vecinos de las comarcas rurales por monopolizar la situación y distribución de las escuelas, no tenían el propósito de favorecer la mejor y mayor difusión de la enseñanza, sino utilizar el alquiler de la casa en que la escuela sería instalada, el sueldo del conserje y las cantidades que pudiera pagar el maestro o la maestra por los gastos en que incurriese.

La dirección técnica de la enseñanza, inspirada por esos principios, mantuvo siempre una escuela sin distinción de índole para todos los niños cubanos. Los cursos de estudios del año 1901, como los de 1902 y 1905, fueron únicos para todas las escuelas públicas, fuesen rurales o no.

El 18 de julio de 1909, se aprobó la primera Ley Escolar por el Congreso de la República, y fue su autor el doctor Ezequiel García, la que borraba toda distinción y diferencia entre las escuelas públicas de la nación; pero la tradición y el hecho consumado eran más decisivamente poderosos que las doctrinas. Bajo la influencia de estos factores, el 5 de marzo de 1914 la Junta de Superintendentes de Escuelas de Cuba dio un Curso y un Plan de Estudio para las escuelas rurales.

Por su parte, la Ley Escolar de 1909 reorganizó las Juntas de Educación, creó las Inspecciones de Distritos y dispuso la estabilidad de los maestros, que hasta entonces tenían que hacer exámenes periódicos para seguir en el disfrute de sus cargos, pues según la Orden Militar No. 368, las Juntas de Educación solo podían contratar a los maestros de su distrito por un año.

Más tarde recibió múltiples modificaciones, como las realizadas por las leyes de 4 de julio de 1911, 19 de julio de 1916, 23 de enero de 1918, así como por la Ley de 18 de abril de 1927, la Ley Orgánica del Poder Ejecutivo de 24 de mayo de 1927, el Decreto-Ley No. 283 de 8 de junio de 1934 y el No. 294 de 13 de junio de 1934. Los elementos anteriores constituyen elementos antecedes al trabajo presentado, pero constituye una valiosa información para comprender la temática analizada a partir del año 1936.

Por el Decreto-Ley No. 620 de 27 de febrero de 1936, se creó un tipo de escuela rural en el que se usarían miembros del Ejército para prestar servicios en la enseñanza primaria, en aquellos lugares 
donde no existiera escuela ni hubiera probabilidad de próxima creación. Por el $\mathrm{n}^{\circ} 707$ se fundó el Instituto Cívico-Militar con la finalidad de combatir el analfabetismo, mejorar las condiciones de vida del campesinado y orientar su vocación, preparándole para su cooperación al mejor desenvolvimiento de la actividad social, para proteger y educar a la niñez desvalida huérfana de padres campesinos, obreros, policías y alistados del Ejército y de la Marina Constitucional que hayan muerto con motivo u ocasión de su trabajo o servicio. Al objeto de sufragar los gastos que las escuelas CívicoMilitares irrogarían, se estableció el impuesto de 9 centavos a cada saco de azúcar elaborado en la República, por medio de la Ley del 30 de diciembre de 1936. Toda esta organización CívicoMilitar integraba una institución de más vastas proyecciones que se denominaba Consejo Corporativo. ${ }^{\mathrm{i}}$

\section{Relación escuela rural-campesinado cubano}

Al enjuiciar la escuela rural habanera como la causa determinante de toda la situación de la vida campesina en este territorio, de la cual se deriva ineludiblemente, en sus más importantes aspectos, la del país entero, surge tan espontánea como impositiva la pregunta:
¿Podría la escuela rural habanera de 1936 a 1952 ser capaz de afrontar y resolver los complejos problemas que planteaba la población campesina?

Los problemas que emergen de la población campesina, no se podían resolver enseñando a leer y a escribir a los niños, a la manera colonial. La escuela rural de aula única, instalada, por lo general, tenían un moblaje inadecuado y su ambiente de incomprensión y de penuria, daban la sensación de algo destinado a reafirmar la indolencia, la apatía y el abandono que acusan todas las cosas de los campos habaneros.

La escuela rural habanera, de acuerdo con los postulados de la Pedagogía, no tenía medios de enseñanzaaprendizaje para impartir una educación creadora de hábitos que elevaran al campesino sobre la sociedad en que vivía, determinando su progreso; ni para impedir que continuara siendo, en casi todas sus manifestaciones, una negación de la civilización y hasta de los más elementales sentimientos humanitarios, y menos para conjurar el resultado adverso, pero inevitable, de dejar en el alma de los jóvenes campesinos, con la noción del saber.

Así, después de más de treinta años de funcionar la escuela pública rural desde el nacimiento de la República Neocolonial 
(1902), en los campos habaneros subsistían inalterable el bohío de yaguas y guano. En la familia campesina, se desconocía la higiene infantil y los gérmenes de enfermedades se encontraban en los niños. La vivienda rural continuaba privada de servicios que le eran indispensables; se mantenían aislados, cultivando el pedazo de tierra, que apenas subviene a sus necesidades, sin acertar y adquirir hábitos de cooperación y de mutualidad, fuera de las cobijas de sus casas.

\section{Problemas y dificultades de la escuela rural habanera}

La escuela rural habanera tenía sus problemas propios y exclusivos creados unos, por el medio ambiente, otros por errores y vicios, que eran urgentes eliminar para ponerla en aptitud de evolucionar hacia el cumplimiento pleno de las finalidades arduas y premisas que le estaban señaladas.

El reclutamiento del magisterio rural fue para la escuela rural, no un problema ni una dificultad, sino un verdadero escollo, lo que condujo entre otros factores a la existencia de escuelas de aula única, sin poder realizar la agrupación de las aulas, la que permitía la división del trabajo escolar.

Al agrupar las aulas, además del trabajo escolar, propiamente dicho, podían ser mejor atendidas, por la acción conjunta de los maestros, las actividades extraescolares en el seno de la comunidad rural, las cuales eran indispensables, porque completan la obra de la escuela, haciéndola alcanzar toda su trascendencia.

En la escuela rural que se acentuaba la incorporación a ella de maestros principiantes para que se adiestraran antes de pasar a servir en las escuelas de los centros urbanos, a las que eran prontamente trasladados los que revelaban buenas prácticas. La consecuencia obligada era que las escuelas rurales no tendrían maestros aptos y entusiastas que las dirigieran, salvo aquellos que el transcurso de su adiestramiento no lograba formar una familia en la comunidad rural, casos estos muy excepcionales.

Al respecto el maestro Felipe (1931) con más de diez años de experiencia rural expresó:

Conozco 25 maestros habilitados pertenecientes a un distrito escolar habanero, de los cuales 21 son maestros rurales y los otros son suplentes en la cabecera del distrito. Este es un hecho repetido en todo el país y que confirma que a las escuelas rurales se destinan los maestros menos reputados.

La inmensa mayoría de los maestros rurales del contexto analizado (19361952), como consecuencia inevitable, estaban integrado por jóvenes que dominan las materias que aprendieron en las 
Escuelas Normales, pero les gustaba enseñar aquella materias en que se sentían más preparados y que les habían gustado más y descuidaban las demás. En su mayoría carecen de preparación para las cuestiones privativas del campo y de paciencia y hábitos para ellos y no estiman que fuera necesario adquirirlas, porque el ansia de ser maestros urbanos los domina, los hacía considerarse como maestros transitorios en el lugar en que trabajan, aunque siempre estuvo en menor cuantía, el maestro de verdadera vocación que se impuso ante todas esas dificultades y supo enfrentar los obstáculos que le imponía la realidad del campesinado habanero.

La procedencia urbana de la mayoría de los maestros; su falta de afinidad con la rusticidad de la cuestión del campo y su desconocimiento de los intereses y de la significación de la comunidad rural, los conducía a alentar en sus estudiantes la aspiración de ser hombres urbanos, por lo que se impute a la escuela rural el haber procesado y favorecido el éxodo campesino con todos sus funestos derivados.

El éxodo campesino había determinado la despoblación de los campos habaneros, según datos del período (19301940) mientras la población urbana había aumentado en más de un treinta por ciento, la población rural solo acusa un aumento de un veinte por ciento.

Sobre el trascendental problema de la escuela rural cubana, Felipe Correoso del Risco realizó una investigación con un núcleo importante de estudiantes campesinos de unas cuantas escuelas rurales de La Habana, en ella se aplicó una encuesta sobre los intereses profesionales futuros donde el 75.41 por ciento de los niños que contestaron, escogieron ocupaciones que no eran campesinas, habiendo mostrado preferencia por las de abogado, albañil, barbero, carpintero, comerciante, conductor de trenes, chofer, dibujante, empleado de banco, farmacéutico, ingeniero, maestro, maquinista, marinero, mecánico, mecanógrafo, médico, oficinista, panadero, tenedor de libros.

El 24,59 por ciento de los niños que contestaron, fueron los que optaron por ocupaciones rústicas, evidenciando interés por las de agricultor, calero, carretero, labrador, lechero, pesador de caña, ganadero, sabanero, y vaquero, obteniendo que la ocupación campesina de los padres influyó poco, pues no subió del 12 por ciento, pero se pronunció más la influencia del medio, pues los niños de la región en que se cultivan vegas, por ejemplo, son los que manifiestan el deseo de ser vegueros, y 
los de las zonas de las fincas azucareras, el de tener trabajos propios de los ingenios.

Las influencias políticas habían contribuido a crear y a empeorar el problema de la situación de las escuelas rurales habaneras. En muchos casos las escuelas rurales no se habían situado, teniendo en cuenta la densidad de la población escolar y a lo accesible que para esta sea el lugar en que la escuela quedara establecida, sino a otras consideraciones muy diferentes. Ante tal situación el maestro Henrique, Enrique (1948) expresó: La escuela rural cubana no existe.

En la primera mitad del siglo $\mathrm{XX}$, existían según criterios cuantitativos de varios autores como, Correoso (1931), Quintana (1948) y Enrique (1948), unas 4200 aulas rurales en La Habana. Al respecto Enrique (1948) planteó: Sabiendas de que nos quedamos cortos, que hacen falta 8000 .

En muchos lugares eran los mismos campesinos los que se ofrecían para hacer las escuelas y a veces las hacían como podían y con sus propios recursos. Según el censo de 1943, de los 4778583 habitantes que tenía la República 2607490, o sea el 54,5 por ciento habitaban en las ciudades: y 2171095 o sea el 45,5 por ciento en los campos. El porcentaje total de niños de 5 a 13 años de edad es de 21.1 por ciento, o sea 100281. El Anuario Estadista o Escolar. Avance $\mathrm{n}^{\circ}$. 1, de 1946, del Ministerio de Educación calcula la población escolar, de 6 a 14 años, de 1009133, de estos 458100 son niños campesinos, de ellos varones el 10.25 por ciento y hembras el 10,75. Mientras los 550181 niños de 5 a 13 años, de las ciudades, disponían de 9395 aulas - sin contar las de las escuelas privadas - los niños campesinos tienen que contentarse con 4061 aulas. La justa proporción seria de 7622 aulas para los niños campesinos.

La construcción y la ubicación de las escuelas rurales son en extremo defectuosas. La gran mayoría de los locales estaban en mal estado, y muchos en condiciones desastrosos. Muchos niños campesinos tenían que dar clases en casas de tabaco, en simples bohíos, en locales deteriorados donde el agua y el viento tenían libre acceso.

Otra extraña fatalidad era que las escuelas estaban situadas lejos de los niños, en el interior de las fincas no había escuelas. En ocasiones los campesinos querían enviar sus hijos a la escuela, pero temían por las largas distancias que debían recorrer entre maniguas o caminos muy tupidos por las hierbas.

Opinión de una maestra normalista sobre el problema de las escuelas rurales (escuelas campesinas) 
La maestra normalista Lía Sastra Latiera quien cita Enrique (1948) expresó:

Soy una defensora cierta de la idea de que la escuela rural no debe, porque no puede, ser igual a la urbana; y esta opinión mía, en la que no abrigo el más pequeño interés personal, no es nueva en mí, porque así lo expuse, junto con el compañero Fernando Cantío, en el informe que ambos rendimos, en marzo de 1939 a la Comisión que alrededor del problema rural escribimos textualmente: "El Plan y Cursos de Estudios de las escuelas urbanas deben ser distintos a los de los que asumen las rurales, pues sus necesidades son distintas y deben ser también distintas sus aspiraciones.

Contaba la profesora Lía que cuando ella no tenía aún un aula urbana donde impartir sus clases, se dispuso a trabajar en una escuela rural, para reafirmar en ella las creencias y demostrarse que los maestros normalistas difícilmente podían triunfar en el campo, por muchas razones, entre ellas, principalmente, el absoluto desconocimiento de la vida campesina y de sus problemas fundamentales, lo que no les permitía la adaptación a aquel medio tan diferente.

Refiere la profesora que ella como todas las maestras normalistas, desertó de la escuela rural tan pronto pudo, y que la recuerda como un mal sueño, a pesar de que se empeñó en cumplir con su deber mientras le sirvió.

\section{La discriminación del niño rural y la deserción del maestro rural}

Los datos que el Departamento de Estadística del Ministerio de Educación (1949) ofrece sobre la desgraduación escolar, entendiendo por tal cosa el desnivel que existe entre el grado escolar de un alumno y su edad cronológica, muestran que el niño campesino está casi siempre por debajo del grado escolar que le corresponde, en tanto que la graduación de los niños urbanos sigue, más o menos, la línea de la graduación que debían tener, de acuerdo con su edad. Es de destacar, en particular, el pico abrupto que presenta la curva de desgraduación en el primer grado, casi todos los escolares campesinos están en el primer grado.

En cuanto a los maestros, era frecuente la deserción, por lo que aproximadamente representaba que un 20 por ciento de las escuelas rurales habaneras carecían permanentemente de maestros y de su deficiente preparación frente a la tarea que les esperaba en los campos y sin embargo, quizá más que ninguno, el maestro rural debía estar a la altura de su misión, y ser capaz de influir en sus alumnos en muchos sentidos, sobre el medio que lo rodeaba; no podía no podía ser simplemente un enseñador de primeras letras. 
Al respecto el Código de Educación para los Niños del Campo (1938) plantea: Todo niño campesino tiene derecho a tener maestros, inspectores y administradores que conozcan la vida rural y que hayan sido educados y formados para ocuparse efectivamente de los problemas peculiares de las escuelas rurales.

Era un hecho que muchos maestros se rehusaban a trabajar en el campo y que permanecían desempeñando un aula rural durante el tiempo de servicio social, o el suficiente para hacer gestiones en los altos centros políticos y educacionales para conseguir la designación en un aula urbana. En los casos en que estas gestiones no tenían éxitos lo que el maestro hacía era trasladar el aula al sitio que le permitía con mayor facilidad ponerse en comunicación con el centro urbano donde residía y vivía en un constante ir y venir entre su casa y la escuela, atendiendo a esta última las horas que marca, estrictamente, el horario de clases.

El maestro rural, no podía dedicar un solo momento a visitar a los vecinos de la comunidad, a recibirlos en la escuela, a tomar parte en los festivales y actos que realicen las instituciones de la comunidad rural, y como consecuencia, su influencia no podía dejarse sentir en aquel medio. Aunque no en todos las experiencias habaneras fue así, ya que como se ha expresado anteriormente, hubo muy buenos maestros y con excelente experiencias pedagógicas en las aulas rurales de La Habana.

¿Cuáles eran las principales deficiencias del servicio que prestaban los maestros rurales habaneros, aparte de la ya señalada desproporción numérica respecto al magisterio urbano?

Puede decirse que todas las aulas rurales estaban dotadas de maestros, pero no que podían disponer de ellos, con regularidad, los niños campesinos. Esto se debía, al hecho de que, habitualmente, los maestros rurales no residían en la escuela, o en sus vecindades, circunstancia que tiende a traducirse, en mayor o menor grado en irregularidad e ineficiencia, además de acarrear incomodidades y gastos suplementarios a los propios maestros. También resulta de este modo que el maestro permanecía alejado del medio, de las familias de sus alumnos, de los problemas del ambiente que rodeaba a su escuela.

Los maestros rurales habaneros debían convertirse en el alma de la escuela e importante elemento rector en el ambiente social, pasa así a ser guía intelectual y factor social indispensable de la comunidad; en la mayor parte de los casos, no se sentían identificados con esta, por lo que como se explicó anteriormente, 
desertaban una vez concluido su período de adiestramiento y que lograban buscar una plaza en las ciudades, esencialmente ocurría en los casos de los maestros rurales de sexo femenino.

Al maestro rural no se le trata bien. En vez de premiar con gratificaciones extraordinarias a los que cumplían satisfactoriamente en zonas lejanas y a veces aisladas y dejarles entrever la posibilidad de ascensos, mejoras $y$ traslados ventajosos, se les ignoraba.

La política, y aún la politiquería, se imponían por encima de los esfuerzos, la constancia y la conducta intachable era un factor que desanimaba y desmoralizaba a los maestros rurales a inducir a un peligroso relajamiento a los inspectores y a los funcionarios técnicos encargados de supervisar y dirigir. Por otra parte, es preciso aceptar que a muchos de los maestros rurales les faltaban doctrinas educativas adecuadas, y aprendizajes especiales, los que no se les enseñaba en su formación en la Escuela Normal para Maestros de La Habana.

\section{Sobre la procedencia y formación de los maestros rurales}

En su mayoría los maestros de las escuelas rurales procedían de la ciudad y tenían que viajar por largos caminos hasta llegar a las escuelas. No podían alquilar automóviles ya que no les alcanzaba el dinero. A respecto Quintana (1934) expresó: ¡Están tan caros los transportes y es tan corto el sueldo que ganamos en relación con la carestía de la vida!

Los maestros de las escuelas rurales de La Habana se formaban en la Escuela Normal para Maestros de La Habana, donde recibían una adecuada formación, aunque limitada a experiencias urbanas, sin embargo en su mayoría cuando se graduaban debían trabajar en escuelas rurales.

Según el maestro rural Quintana, (1949) no se enseñaban en las clases de Psicología Infantil sobre la conducta del niño campesino, ni a resolver los conflictos sobre las diferencias de edades en una misma clase, al respecto expresó: Nos han dado nociones bastantes confusas por cierto acerca de la medición de la inteligencia, del esfuerzo muscular, de las aptitudes, del valor acerca de cómo debemos reaccionar ante una actitud rebelde o pasiva persistente, de nuestros alumnos.

Desde los testimonios de este maestro rural, existía una formación de maestros ajena de la realidad educativa y de las prácticas de la vida en el campo cubano. Esto hacía que en muchos casos los maestros desmotivados abandonaran las aulas o dedicaran tiempo a prepararse 
en función de las necesidades reales que el contexto de su actuación les exigía o se convertían en desertores y abandonaban el aula rural por una urbana, que desde los estudios realizados fue esta la tendencia que existió.

...por qué decirle a un niño cuyos padres apenas tienen con qué calzarlo $\mathrm{y}$ vestirlo, que existen tractores modernos que permiten roturar la tierra en menos tiempo que con el arado criollo, que el abono químico hace producir mayor rendimiento a las cosechas y que el riego artificial sustituye (muchas veces con ventajas) a la naturaleza, es despertar en ellos un sentimiento de inconformidad con su vida habitual, que forzosamente ha de traducirse en el deseo de abandonarla, ante la impotencia de obtener el remedio.

Ante ese conflicto psicológico se enfrentaron muchos maestros, los que tenían la responsabilidad de resolverlo desde sus enseñanzas, tanto en la clase como en sus charlas con padres y vecinos de la escuela.

La desatención del campo cubano por parte de los gobiernos republicanos, exigía gran esfuerzo de los maestros rurales habaneros, los que sin recibir una preparación sobre los principales conflictos que tendrían que resolver, tenían que enfrentar la situación y aunque no todos, maestros como Julio Quintana y Raúl Ferrer, entre otros, supieron poner en alto el magisterio cubano, prevaleciendo la creatividad y la constante superación e indagación.

Ante la llegada de los maestros a una zona rural, debían identificarse con los vecinos de los alrededores y lograr ser aceptados por los familiares de los futuros alumnos. Era indispensable que para garantizar la asistencia a clases, el maestro se hubiera convertido en uno más entre ellos, sin rasgos de vida de la ciudad. Por estas razones cuando llegaban los maestros, la primera tarea era con los padres, lograr que paulatinamente cambiaran sus concepciones sobre lo que debían aprender sus hijos.

Quintana (1949) expresaba que los guajiros recibía a los maestros con estas palabras: Enseñe a mi hijo a leer, escribir y contar y no se ocupe de otras boberías, que para enseñarle a trabajar estoy yo.

Era de esperar una reacción como esa, ante un desconocido bien vestido que llegaba de la ciudad en un caballo y que quería reclutar a todos los niños, adolescentes y jóvenes de la zona, para enseñarles durante todo el día. Ante tal panorama, los padres apenas el niño aprendía a leer y a escribir lo retiraban de la escuela, para que se dedicara a trabajar en el campo y ayudara con las tareas hogareñas como halar agua, enyugar los bueyes y pastorear el ganado entre otros. 


\section{La llegada del maestro a la zona: sus condiciones de vida y de la escuela. El primer día de clases}

Al llegar los maestros a las escuelas rurales, la imagen que transmitían era de soledad, y aislamiento. Se describen como un cajón de madera con techo de guano que por su aspecto externo parecían llevar bastante tiempo construidas, carecían de un portal en su gran mayoría aunque este era un símbolo de consolidación de la escuela, orientadas en su mayoría hacia el este, lo que facilitaba la luz durante el día, aunque el sol castigaba bastante el interior del aula durante las horas de la mañana. No se percibían árboles a sus alrededores, solo las hierbas a la altura de un metro que apenas se lograba ver las ventanas de la escuela. Difícil panorama para un joven recién graduado de la Escuela Normal para Maestros de La Habana. ${ }^{\text {ii }}$

La primera tarea del maestro era buscar donde dormir ya que viajar era muy costoso y en difíciles condiciones. El maestro debía acercar la escuela esta la comarca (barrio campesino) más cercana donde se hospedaba y desde donde podía viajar con más facilidad en un caballito flaco que compraba. Esta alternativa que fue la más predominante dificultaba el acceso de los alumnos a las escuela, pues el traslado a estas se hacía más lejano y difícil, por lo que los maestros más interesados y astutos acudían a la hospitalidad del campesinado cubano, quienes le ofrecían en su mayoría un cuarto con mal olor y ruidoso. A pesar de la inicial desconfianza que le provocaba el maestro a la familia, paulatinamente se familiarizaban y se ayudaban mutuamente.

En las noches, el maestro alumbrándose con un farol enseñaba a leer y a contar al padre de la familia y así le retribuía el hospedaje que este le ofrecía. Estas acciones propiciaron que los maestros rurales se ganaran el respeto y la confianza de la familia.

La organización de la escuela para comenzar las clases era otra de las tareas que tenían que desarrollar estos maestros, expresaba Quintana (1949):

De adornos no se puede hablar por ahora; las paredes no pueden estar más descuidadas y muestran las huellas de la humedad lo que demuestra las filtraciones del techo; de carteles o colgadizos no se hable... y los pupitres están completamente destruidos. No hay bufetes, ni sillas, ni armario.

Con el apoyo del conserje, quién se ocupaba de buscar ayuda entre los vecinos de la zona y con los mismos alumnos, limpiaban el local, chapeaban los alrededores de la escuela, cobijaban el techo de la escuela de forma que les cubriera del agua lluvia y de las entradas de sol durante la clase. 
Al decir Quintana (1949) sobre la escuela rural: La escuela campesina tiene que ser más que ninguna, una escuela socializada. Hay que desarraigar de la mente del vecindario la idea de que este lugar es algo así como la colina sagrada.

Romper con esas barreras era una de las tareas de los maestros rurales. Se habían ganado el respeto y la consideración de la comunidad. El maestro de la ciudad se había convertido en uno más, ya dominaba las principales costumbres del campo, los procedimientos, la forma de pensar y de actuar de esos humildes hombres. La escuela lista para comenzar, los padres bajo regaños autorizaban la asistencia de sus hijos durante un rato, después debían incorporarse al trabajo en el campo o al pastoreo del ganado.

El primer día de clase era muy difícil, los estudiantes contestaban a medias palabras entre dientes, no levantaban la vista, no miraban a la cara. Los profesores tenían que dedicar los primeros días de clase para el diagnóstico del grupo, para eso utilizaba el cuento y el juego para ganarse su confianza. Aplicaban entrevistas al conserje y a los dueños de la casa donde dormía, de esa forma completaba la información que le permitía conformar los grupos y establecer los horarios. Esto dependía en muchos casos no solo de los programas establecidos sino de las experiencias y la creatividad que cada maestro le impregnaba a su clase.

\section{Conclusiones}

El proyecto educativo de escuelas rurales cívico-militar iniciado por Fulgencio Batista en 1936, significó un avance para la educación en el campo cubano, ya que varias escuelas abrieron sus puestas, otras fueron reparadas, además se promovió durante esos años en el discurso político de las principales autoridades, la preocupación por mejorar las condiciones del campesinado cubano, aunque en la práctica los principales problemas no fueron solucionados. La desatención a las escuelas, la ausencia de maestros y la no preparación adecuada a los futuros maestros rurales, fueron de los principales problemas que enfrentó la escuela rural habanera de 1936 a 1952.

\section{Referencias}

Correoso del Risco, F. (1931). La escuela rural cubana, su evolución y significación histórico-social. La Habana: Editorial Hermes. p. 65.

Correoso del Risco, F. (1931). La escuela rural cubana, su evolución y significación histórico-social. La Habana: Editorial Hermes. p. 68. 
La escuela rural cubana, su evolución y significación histórico-social. La Habana: Editorial Hermes. p. 89.

Henríquez Henríquez, E. (1948). Escuela para los campesinos. La Habana. p. 85.

Henríquez Henríquez, E. (1948). Escuela para los campesinos. La Habana. p. 91.

Quintana Díaz, J. (1934). Por la escuela rural. Ponencia presentada a la junta de Superintendente. p. 6.

Quintana Díaz, J. (1949). Mañana. Memorias de un maestro rural. La Habana. p. 10.

Quintana Díaz, J. (1949). Mañana. Memorias de un maestro rural. La Habana. p. 11.

Quintana Díaz, Julio. M. (1949). Memorias de un maestro rural. La Habana. p. 12.

Quintana Díaz, Julio. M. (1949). Memorias de un maestro rural. La Habana. p. 19.

Quintana Díaz, Julio. M. (1949). Memorias de un maestro rural. La Habana. p. 20.

\footnotetext{
i Los datos que sostienen las informaciones extraídas por el autor se localizan el Decreto Ley No. 620 del 27 de febrero de 1936. Edición extraordinaria número 53 de la Gaceta Oficial del 27 de febrero de 1936. Fondo 24 Ejército. Archivo Instituto de Historia de Cuba.

ii La descripción ofrecida es el resultado de la sistematización realizada por el autor a partir de diversos textos consultados, así como la observación de fotos de las escuelas rurales habaneras del período abordado.
}

Recebido em: 15/12/2016

Aprovado em: 10/01/2017

Publicado em: 19/04/2017

Como citar este artigo / How to cite this article / Como citar este artículo:

APA:

Castillo, Y. L. (2017). Las escuelas rurales de La Habana: un problema sin resolver en la República Neocolonial entre 1936 y 1952. Rev. Bras. Educ. Camp., 2(1), 184-203.

DOI:

http://dx.doi.org/10.20873/uft.2525-

4863.2017v2n1p184

\section{ABNT:}

CASTILLO, Y. L. Las escuelas rurales de La Habana: un problema sin resolver en la República Neocolonial entre 1936 y 1952. Rev. Bras. Educ. Camp., Tocantinópolis, v. 2, n. 1, p. 184-203, 2017. DOI: http://dx.doi.org/10.20873/uft.25254863.2017v2n1p184 